\title{
Risk factors of poor prognosis in patients with pyrrolidine alkaloids induced hepatic sinusoidal obstruction syndrome after transjugular intrahepatic portosystemic shunt
}

\author{
Transjugular intrahepatic portosystemic shunt in patients with sinusoidal obstruction \\ syndrome, etiology matters
}

\author{
Marta Magaz $^{1,2,3} \cdot$ Juan Carlos García-Pagán ${ }^{1,2,3}$ (1)
}

Received: 19 January 2021 / Accepted: 22 February 2021 / Published online: 17 March 2021

(c) Asian Pacific Association for the Study of the Liver 2021

\begin{tabular}{|c|c|}
\hline \multicolumn{2}{|c|}{ Abbreviations } \\
\hline SOS & Sinusoidal obstruction syndrome \\
\hline HSCT-SOS & $\begin{array}{l}\text { Hematopoietic stem cell transplantation and } \\
\text { chemotherapy }\end{array}$ \\
\hline PAs & Pyrrolidine alkaloids \\
\hline TIPS & $\begin{array}{l}\text { Transjugular intrahepatic portosystemic } \\
\text { shunt }\end{array}$ \\
\hline PT & Prothrombin time \\
\hline
\end{tabular}

Sinusoidal obstruction syndrome (SOS) is a relatively rare but potentially life-threatening complication that has been traditionally associated in Europe with hematopoietic stem cell transplantation and chemotherapy (HSCT-SOS), while in China is more frequent after ingestion of pyrrolidine alkaloids (PAs), main component of Chinese medicinal herbs or herbal and dietary supplements [1]. Among the described functional changes associated with SOS are toxic damage to endothelial cells and zone III hepatocytes [2]. The sloughed sinusoidal lining cells may block sinusoidal flow and these hemodynamic changes combined may cause progressive post-sinusoidal portal hypertension, abdominal distension,

Juan Carlos García-Pagán

jcgarcia@clinic.cat

1 Barcelona Hepatic Hemodynamic Laboratory, Liver Unit, Hospital ClínicInstitut de Investigacions Biomèdiques August Pi I Sunyer (IDIBAPS), University of Barcelona, Villarroel 170, 08036 Barcelona, Spain

2 CIBEREHD (Centro de Investigación Biomédica en Red Enfermedades Hepáticas Y Digestivas), Barcelona, Spain

3 Health Care Provider of the European Reference Network On Rare Liver Disorders (ERN-Liver), Paris, France jaundice, ascites, and might result in multi-organ dysfunction and death.

Treatment of PAs-SOS is based in a stepwise strategy. Administration of reduced glutathione and ademetionine for liver protection, anticoagulation and diuretics are the first step [3-5]. Then a careful monitoring of ascites and jaundice should be performed during this conservative stage, and a worsening ascites and jaundice should prompt intervention with transjugular intrahepatic portosystemic shunt (TIPS) to avoid progression to severe SOS [5, 6]. Mortality in patients with PAs-SOS in whom the initial therapy fails is high, around $40 \%$ in recent cohorts [5, 7]. This poor prognosis is markedly improved if TIPS is early applied after failure of initial therapy [5].

However, there is still a number of patients with severe PAs-SOS that despite receiving a TIPS will die. The study by Jiangqiang et al. [8] published in the current issue of Hepatology International retrospectively evaluated the outcome of a group of 116 patients with severe PAs-SOS that received a TIPS with the aim to identify predictive factors of poor prognosis after TIPS. The authors of the study found a prothrombin time $(\mathrm{PT})>17.85 \mathrm{~s}$ at baseline and a serum bilirubin $>9 \mathrm{mg} / \mathrm{dl} 5$ days after TIPS as independent predictors of poor survival after TIPS. Six-month survival was around $95 \%$ in patients without risk factors, $80 \%$ in patients with only one risk factor and was markedly reduced to less than $35 \%$ survival in patients with both risk factors. According to these data, the authors recommend caution to consider TIPS in the presence of these risk factors. We must congratulate the authors for being able to recruit such a huge number of patients with a PAs-SOS treated with TIPS and for the effort done to better define prognosis in these patients.

Nevertheless, the authors include in the model variables occurring once the TIPS is already performed (bilirubin at 
post-TIPS day 5). Therefore, although this parameter will help to recommend caution in the post-TIPS follow-up, it will not help when discussing whether a step further to TIPS is worthy to be performed. Unfortunately, we do not know whether the predictive value of baseline PT, instead of including 5-day parameters, would have been also improved by adding in the analysis exclusively other baseline pre-TIPS variables. In addition, including post-TIPS variables does not allow to test the prognostic score in patients not receiving TIPS and therefore does not allow to know how the prognosis would have been, in this same population of patients, if a TIPS would have not been placed. We must be aware that we are dealing with a PAs-SOS population in whom the initial treatment fails, then, it could be that the outcome of these patients would have been even worse if a TIPS had not been performed. Indeed, in other different scenarios, such as the use of pre-emptive TIPS for treatment of high-risk variceal bleeding, use of TIPS, even in patients with characteristics associated with poor post-TIPS prognosis, have a better prognosis than in those with the same clinical characteristics in whom TIPS was not done [9]. For that purpose, it would have been interesting to know prognosis in those patients, that although TIPS was indicated, they refused it. Comparing patients with baseline poor prognostic indicators treated with TIPS or not would have answered the relevant question, if poor prognostic criteria should lead to discard TIPS performance or not.

On the other hand, although the number of PAs-SOS patients treated in this cohort with TIPS is a large sample, the number of patients that present a poor prognosis postTIPS is small, a fact that reduces the strength and potential reproducibility of the study findings. The study by Jiangqiang et al. [8] provides a rationale to extend research in this field, encouraging the launch of multicentric studies including a large number of patients treated with or without TIPS as the best way to answer these questions.

An additional relevant question is whether we can extrapolate the results of this study to other SOS etiologies. The present study, reported 1- and 5-year survival of PAsSOS patients treated with TIPS considerably higher than the reported in patients receiving TIPS because of HSCTSOS $[4,10]$. This may be associated, as the authors well described in the manuscript, to the fact that patients with PAs-SOS usually are, contrary to HSCT-SOS patients, in a previous healthy condition [6]. In addition, it is worthy to mention that in the current study, TIPS was placed very early after PAs-SOS was diagnosed and the initial therapy applied considered to have failed. Usually in the HSCT-SOS setting, TIPS is applied later and usually in a more deteriorated status and associated with a very poor outcome. Therefore, it is highly likely that the early performance of TIPS markedly may influence the outcome. A recent small cohort of patients with severe HSCT-SOS would support the early application of TIPS in severe cases failing to initial therapy. Indeed, in this cohort, TIPS was applied in a mean of 4 days after SOS diagnosis achieving a $100 \%$ of 100 -day survival, maintaining after TIPS treatment with defibrotide [11]. Whether earlyTIPS treatment in other etiologies of severe SOS could also improve post-TIPS prognosis is still an open question that requires further assessment.

Acknowledgements We are indebted to administrative support by Oliva Ros Fargas.

Financial support statement This study was supported by the Ministry of Education and Science (SAF-2016-75,767-R). Instituto de Salud Carlos III (ISCIII) \& Fondo Europeo de Desarrollo Regional (FEDER): (PIE15/00,027 and PI18/00,205). Centro de Investigación Biomédica en Red de Enfermedades Hepáticas y Digestivas (CIBERehd), funded by the Instituto de Salud Carlos III. Marta Magaz is a recipient of a Río Hortega grant from Instituto de Salud Carlos III, Spain.

\section{Declarations}

Conflict of interest JCGP advisory for GORE, Cook and Shionogi. Marta Magaz declares no conflicts of interest that pertain to this work.

\section{References}

1. Yang X-Q, Ye J, Li X, Li Q, Song Y-H. Pyrrolizidine alkaloidsinduced hepatic sinusoidal obstruction syndrome: pathogenesis, clinical manifestations, diagnosis, treatment, and outcomes. World J Gastroenterol. 2019;25:3753-63.

2. DeLeve LD, Ito Y, Bethea NW, McCuskey MK, Wang X, McCuskey RS. Embolization by sinusoidal lining cells obstructs the microcirculation in rat sinusoidal obstruction syndrome. Am J Physiol Liver Physiol. 2003;284:G1045-52.

3. Peng C, Zhang X, Zhang F, Liu L, Shao Y, Xiang X, et al. Clinical efficacy and safety of anticoagulation therapy for Pyrrolizidine alkaloids-induced hepatic sinusoidal obstruction syndrome: a retrospective multicenter cohort study. Eur J GastroenterolHepatol. 2020;32:1168-78.

4. Zenz T, Rössle M, Bertz H, Siegerstetter V, Ochs A, Finke J. Severe veno-occlusive disease after allogeneic bone marrow or peripheral stem cell transplantation-role of transjugular intrahepatic portosystemic shunt (TIPS). Liver. 2001;21:31-6.

5. Zhou C-Z, Wang R-F, Lv W-F, Fu Y-Q, Cheng D-L, Zhu Y-J, et al. Transjugular intrahepatic portosystemic shunt for pyrrolizidine alkaloid-related hepatic sinusoidal obstruction syndrome. World J Gastroenterol. 2020;26:3472-83.

6. Zhuge YZ, Wang Y, Zhang F, Zhu CK, Zhang W, Zhang M, He Q, Yang J, He J, Chen J, Zou XP. Clinical characteristics and treatment of pyrrolizidine alkaloid-related hepatic vein occlusive disease. Liver Int. 2018. https://doi.org/10.1111/liv.13684.

7. Wang Y, Qiao D, Li Y, Xu F. Risk factors for hepatic veno-occlusive disease caused by Gynura segetum: a retrospective study. BMC Gastroenterol. 2018;18:156.

8. Jianggiang X, Jingjing T, Han Z, et al. Risk factors of poor prognosis in patients with pyrrolidine alkaloid-induced hepatic sinusoidal obstruction syndrome after transjugular intrahepatic portosystemic shunt. Hepatol Int. 2021 (In press).

9. Nicoară-Farcău O, Han G, Rudler M, Angrisani D, Monescillo A, Torres F, et al. Effects of early placement of 
transjugularportosystemic shunts in patients with high-risk acute variceal bleeding: a meta-analysis of individual patient data. Gastroenterology. 2021;160(193-205):e10.

10. Azoulay D, Castaing D, Lemoine A, Hargreaves G, Bismuth H. Transjugular intrahepatic portosystemic shunt (TIPS) for severe veno-occlusive disease of the liver following bone marrow transplantation. Bone Marrow Transpl. 2000;25:987-92.

11. Gómez-Centurión I, Bailén R, Oarbeascoa G, Muñoz C, Luque AÁ, Boyra ME, et al. Transjugular intrahepatic portosystemic shunt for very severe veno-occlusive disease/sinusoidal obstruction syndrome (VOD/SOS) after unmanipulatedhaploidentical hematopoietic stem cell transplantation with posttransplantation cyclophosphamide. Biol Blood Marrow Transpl. 2020;26:2089-97.

Publisher's Note Springer Nature remains neutral with regard to jurisdictional claims in published maps and institutional affiliations. 